\title{
Ox-LDL increases OX40L in endothelial cells through a LOX-1-dependent mechanism
}

\author{
Q. Dong, R. Xiang, D.Y. Zhang and S. Qin \\ Department of Cardiology, The First Affiliated Hospital, Chongqing Medical University, Chongqing, China
}

\begin{abstract}
Oxidative low-density lipoprotein (Ox-LDL) is a key risk factor for the development of atherosclerosis, and it can stimulate the expression of a variety of inflammatory signals. As a new and highly sensitive inflammation index, OX40L may be a key to understanding the mechanisms that regulate interactions between cells within the vessel wall and inflammatory mediators during the development of atherosclerosis. To investigate whether Ox-LDL regulates OX40L expression through an oxidized LDL-1 receptor (LOX-1)-mediated mechanism, we investigated the effect of different concentrations of Ox-LDL (50, 100, $150 \mu \mathrm{g} / \mathrm{mL}$ ) on endothelial cell proliferation and apoptosis. Stimulation with Ox-LDL increased OX40L protein 1.44-fold and mRNA 4.0-fold in endothelial cells, and these effects were inhibited by blocking LOX-1. These results indicate that LOX-1 plays an important role in the chronic inflammatory process in blood vessel walls. Inhibiting LOX-1 may reduce blood vessel inflammation and provide a therapeutic option to limit atherosclerosis progression.
\end{abstract}

Key words: Atherosclerosis; Ox-LDL; LOX-1; OX40/OX40L; Polyinosinic acid

\section{Introduction}

The pathogenesis of atherosclerosis and preventive countermeasures are important therapeutic considerations. The inflammatory response plays a very important role in coronary atherosclerotic heart disease (1). Atherosclerosis is a chronic disease characterized by an inflammatory hyperplasic response elicited by injury to vascular endothelial and smooth muscle cells in the artery wall, promoting local accumulation of oxidative lowdensity lipoprotein (Ox-LDL) (2-4). Accumulated Ox-LDL can stimulate the expression of a variety of inflammatory signals through activation of the lectin-like oxidized lowdensity lipoprotein-1 receptor (LOX-1) (5).

A new and highly sensitive inflammation index, OX40L, is present in almost all of the cells involved in atherosclerosis, including endothelial cells, lymphocytes, macrophages, and smooth muscle cells (6). Clinical studies have shown that levels of soluble OX40L in blood plasma are significantly higher in patients with acute coronary syndrome or stable angina than in the normal population. This suggests that OX40L serves as a key protein regulating the interaction between different cells in vascular tissue and inflammatory mediators in the development of atherosclerosis. In addition, OX40L has a very high sensitivity as a marker, indicating that it may be a valuable risk assessment factor for the development and progression of atherosclerosis (7).
During the progression of atherosclerosis, endothelial injury and $O x-L D L$ accumulation in the intima are critical initiating events. Moreover, Ox-LDL plays a role in regulation of the biological function of cells by stimulating cell membrane receptor-mediated endocytosis. Ox-LDL receptors include scavenger receptors $A 1$ and $A 2$, LOX-1, CD36, and CD68 (8). LOX-1, which was first described by Sawamura et al. (9) in 1997, mediates diverse biological effects induced by Ox-LDL stimulation of endothelial cells (10), including phagocytosis of apoptotic and aging endothelial cells and activation of diverse signaling pathways of inflammation $(11,12)$. For example, LOX-1 stimulates the upregulation of CD40 and CD40L, which are associated with inflammation in endothelial cells (13). Polyinosinic acid (Poly I) is a LOX-1 receptor antagonist, but only in combination with Ox-LDL (14). Poly I also inhibits vascular inflammation stimulated by Ox-LDL and thus delays the formation and development of atherosclerosis.

Because the influence and function of Ox-LDL in the OX40/OX40L signaling pathway is unclear, the purpose of this study was to clarify whether Ox-LDL is involved in the OX40/OX40L signaling pathways using an in vitro model of endothelial cell injury. In addition, we investigated the underlying mechanisms involved in order to provide a new theoretical basis and targets for drug therapy for prevention and treatment of atherosclerosis.

Correspondence: S. Qin, Department of Cardiology, The First Affiliated Hospital, Chongqing Medical University, 1 Youyi Road, Chongqing 400016, China. Fax: +86-23-8901-1429. E-mail: clb436@126.com

Received November 14, 2012. Accepted June 7, 2013. First published online September 16, 2013. 


\section{Material and Methods}

\section{Cell lines and reagents}

Human umbilical vein endothelial cells (HUVECs) were obtained from Chongqing Medical University (Chongqing, China) and cultured in RPMI-1640 (Gibco, USA) supplemented with $10 \%$ fetal bovine serum (Gibco). For Ox-LDL injury, HUVECs were treated with various concentrations of Ox-LDL (XieSheng Bio, China) and Poly I (Santa Cruz, USA) for $24 \mathrm{~h}$. Cells were cultivated in a humidified atmosphere with $5 \% \mathrm{CO}_{2}$ at $37^{\circ} \mathrm{C}$.

\section{Cell proliferation assays}

The cell counting kit (CCK-8, Beyotime Institute of Biotechnology, China) assay was used to determine cell proliferation. Increasing concentrations of Ox-LDL (50, 100 , and $150 \mu \mathrm{g} / \mathrm{mL}$ ) were added to cell cultures, which were then incubated for $24 \mathrm{~h}$. Absorbance was detected with a microplate reader at a wavelength of $450 \mathrm{~nm}$ using a 96-well multichannel auto reader (Biotech Instruments, USA). The percentage inhibition of cell proliferation was determined by comparing the absorbance of treated with untreated controls as follows: Inhibition $(\%)=[1-($ A of the experimental sample/A of the control)] $\times 100 \%]$.

\section{Assessment of cell cycle and apoptosis}

Proliferating HUVECs were serum-starved overnight and treated with Ox-LDL $(100 \mu \mathrm{g} / \mathrm{mL})$ in complete media for $24 \mathrm{~h}$. Following treatment, the cells were harvested and fixed in $70 \%$ ice-cold ethanol. The percentages of cells in the $G_{0} / G_{1^{-}}, S-, G_{2^{-}}$and M-phases were quantitated by flow cytometry.

The extent of apoptosis was evaluated by Annexin-V staining. HUVECs were incubated in the presence of OxLDL $(100 \mu \mathrm{g} / \mathrm{mL})$ for $24 \mathrm{~h}$ and stained with Annexin-Vfluorescein isothyocyanate (FITC) and propidium iodide (PI). Samples were analyzed by flow cytometry. The data
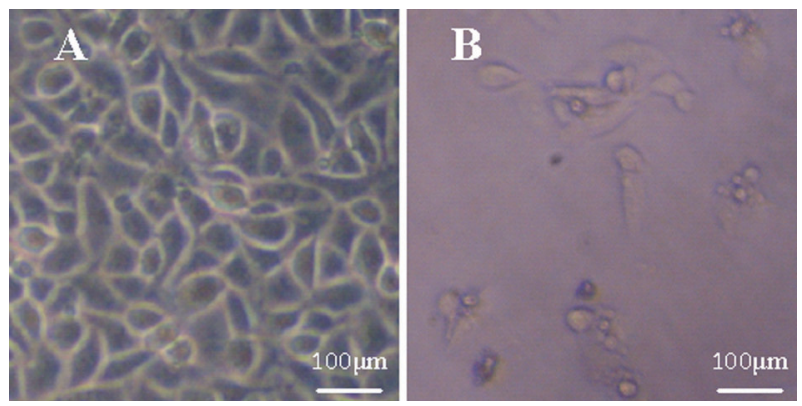

Figure 1. HUVEC shape by inverted microscopy. A, Control HUVECs showing normal adherent growth with a spindle or pentagonal shape. $B$, HUVECs incubated with Ox-LDL $(150 \mu \mathrm{g} /$ $\mathrm{mL}$ ) for $24 \mathrm{~h}$. The cells are shrunken, with an increased rate of cell apoptosis indicated by an increased formation of apoptotic bodies. HUVECs: human umbilical vein endothelial cells; OxLDL: oxidative low-density lipoprotein. shown are representative of at least three independent experimental sets.

\section{Immunoblotting}

HUVECs were plated in a culture flask 1 day before the experiment. The cells were then incubated for $24 \mathrm{~h}$ under the following conditions: a) no Ox-LDL, b) $100 \mu \mathrm{g} / \mathrm{mL}$ Ox-LDL, or c) $250 \mu \mathrm{g} / \mu \mathrm{L}$ Poly I plus $100 \mu \mathrm{g} / \mathrm{mL}$ Ox-LDL (14). The cells were washed three times with ice-cold PBS, lysed with RIPA lysis buffer (Beyotime) and placed on ice for $30 \mathrm{~min}$. Proteins were separated by SDS-PAGE $(12 \%$ gels) and subsequently transferred to a PVDF membrane (Millipore, USA). The membrane was blocked with 5\% BSA in Tris-buffered saline and Tween-20 (10 mM Tris, pH 7.5, $140 \mathrm{mM} \mathrm{NaCl}, 0.05 \%$ Tween-20) for $2 \mathrm{~h}$ at room temperature. A rabbit polyclonal antibody against OX40L (1:1000, Santa Cruz) and a rabbit polyclonal antibody against LOX-1 (1:1000, Abcam, Hong Kong) were used as the primary antibodies, and horseradish peroxidase-conjugated goat anti-rabbit lgG was used as a secondary antibody. BeyoECL Plus (Beyotime) was used for antibody detection according to the manufacturer's instructions.

\section{Immunocytochemistry}

Following the same stimulation conditions described above, HUVECs were fixed in $4 \%$ paraformaldehyde for $20 \mathrm{~min}$, and washed three times in PBS. A rabbit polyclonal primary antibody against OX40L (1:1000, Santa Cruz) was added at $4{ }^{\circ} \mathrm{C}$ overnight. Cells were washed three times in PBS and incubated with the blocking solution, which included FITC-conjugated goat anti-rabbit IgG (CWBio, China), at $37^{\circ} \mathrm{C}$ for $2 \mathrm{~h}$. Cells were washed three times in $\mathrm{PBS}$ and incubated with $\mathrm{PI}$ at $37^{\circ} \mathrm{C}$ for $5 \mathrm{~min}$. Cells were observed with a laser confocal microscope, and the average fluorescence value of eight cells from a random selection was calculated. All images were acquired under identical conditions from a microscope image system.

\section{RNA isolation and real-time qPCR}

Total RNA was isolated from cultured HUVECs using RNAiso Plus (Takara, Japan) according to the manufac-

Table 1. Anti-proliferation effect of Ox-LDL in human umbilical vein endothelial cells.

\begin{tabular}{lcccc}
\hline & \multicolumn{4}{c}{ Ox-LDL $(\mathrm{mg} / \mathrm{L})$} \\
\cline { 2 - 5 } & 0 & 50 & 100 & 150 \\
\hline $\mathrm{A}_{450}$ & $0.96 \pm 0.06$ & $0.81 \pm 0.04^{*}$ & $0.68 \pm 0.03^{*}$ & $0.59 \pm 0.02^{*}$ \\
$\begin{array}{l}\text { Inhibition } \\
\text { ratio }^{\mathrm{a}}\end{array}$ & - & $16.0 \%$ & $29.2 \%$ & $38.7 \%$ \\
\hline
\end{tabular}

Data are reported as mean \pm SD cell proliferation inhibition ratio for $\mathrm{n}=5$ experiments. $\mathrm{A}_{450}$, absorption at $450 \mathrm{~nm}$. ${ }^{\text {a Percentage of }}$ decrease in proliferation rate compared with untreated control group. Ox-LDL: oxidative low-density lipoprotein. ${ }^{*} \mathrm{P}<0.05$ compared with the control group ( $t$-test). 

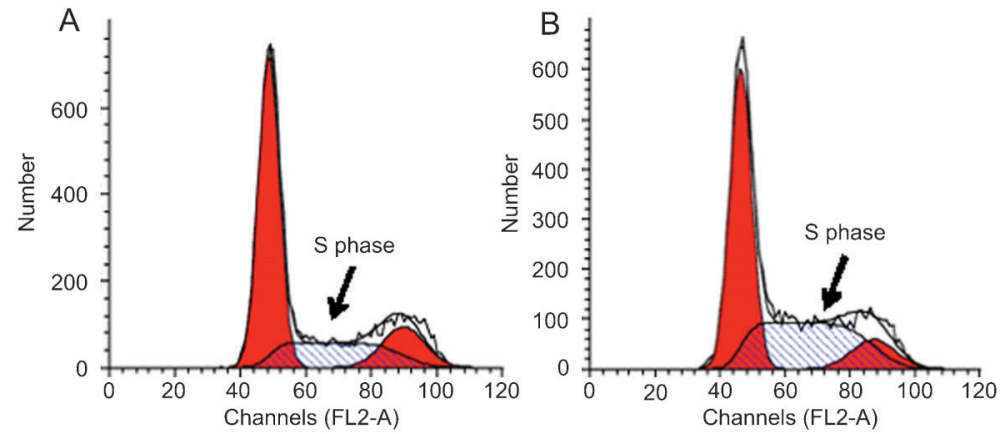

Figure 2. $A$, Cell cycle of normal HUVECs. $B$, HUVECs incubated with Ox-LDL $(100 \mu \mathrm{g} / \mathrm{mL})$ for 24 h. HUVECs: human umbilical vein endothelial cells; Ox-LDL: oxidative low-density lipoprotein.
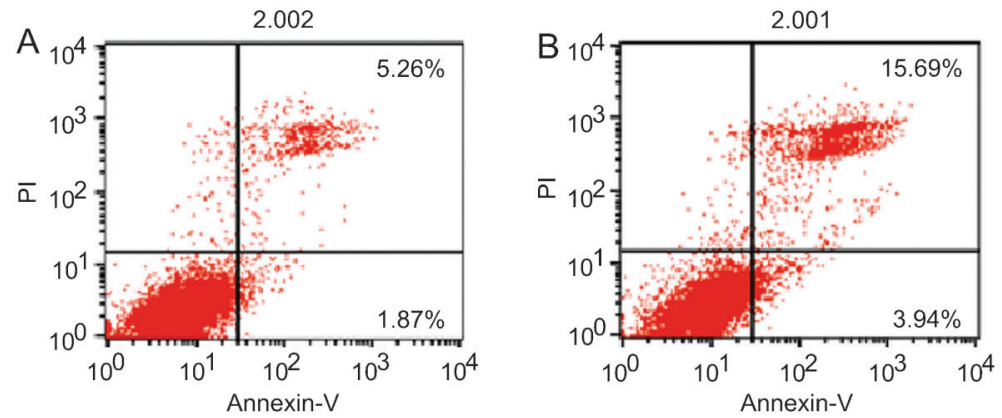

Figure 3. A, Apoptosis rates of normal HUVECs. $B$, HUVECs incubated with Ox-LDL $(100 \mu \mathrm{g} / \mathrm{mL})$ for $24 \mathrm{~h}$. Rates increased from $5.26 \%$ in control to $15.69 \%$ in the Ox-LDL-treated cells. HUVECs: human umbilical vein endothelial cells; Ox-LDL: oxidative low-density lipoprotein; PI: propidium iodide. turer's instructions. First-strand complementary DNA (cDNA) was synthesized using oligo-dT primers and M-MLV reverse transcriptase (Takara). Quantitative PCR was performed with SYBR green PCR Master Mix (Takara). The following primers were used to amplify OX40L CDNA: sense: 5'-CGGGCAGGTCTACTTTGGAG-3' and antisense: 5'-CAGGTCACTGTCCCAGCATC-3'; $\beta$-actin cDNA: sense: 5'-CCACGAAACTACCTTCAACTCC-3' and antisense: 5'GTGATCTCCTTCTGCATCCTGT-3'. PCR was performed using the following PCR conditions: denaturing at $95^{\circ} \mathrm{C}$ for $30 \mathrm{~s}$, annealing at $60^{\circ} \mathrm{C}$ for $20 \mathrm{~s}$, and elongation at $70^{\circ} \mathrm{C}$ for $10 \mathrm{~s}$. The mRNA levels of OX40L were normalized to $\beta$-actin.

\section{Statistical analysis}

Data were reported as means \pm SD using SPSS 17.0. Comparative data were analyzed by multivariate analysis,

Table 2. Changes in the cell cycle and apoptosis rates after human umbilical vein endothelial cells were treated with $100 \mu \mathrm{g} /$ $\mathrm{mL}$ Ox-LDL for $24 \mathrm{~h}$.

\begin{tabular}{cccrc}
\hline Group & \multicolumn{3}{c}{ Cell cycle } & \multirow{2}{*}{$\begin{array}{c}\text { Apoptosis } \\
\text { rate (\%) }\end{array}$} \\
\cline { 2 - 4 } & $\mathrm{G}_{0} / \mathrm{G}_{1}$ & $\mathrm{~S}$ & \multicolumn{1}{c}{$\mathrm{G}_{2} / \mathrm{M}$} & \\
\hline Control & $60.4 \pm 1.1$ & $25.6 \pm 1.5$ & $13.9 \pm 1.5$ & $8.1 \pm 0.9$ \\
Ox-LDL & $55.1 \pm 3.8$ & $35.6 \pm 2.9^{*}$ & $9.3 \pm 0.8$ & $15.6 \pm 3.8^{*}$ \\
\hline
\end{tabular}

Data are reported as means $\pm S D$ for $n=3$ experiments. Ox-LDL: oxidative low-density lipoprotein. ${ }^{*} \mathrm{P}<0.05$ compared to the control group (t-test). and the Student $t$-test was used for paired data. A value of $\mathrm{P}<0.05$ was considered to be statistically significant.

\section{Results}

\section{Ox-LDL inhibited proliferation of HUVECs}

Ox-LDL, which plays a key role in the endothelial injury response, inhibited HUVEC proliferation. There was a decrease in cell number and an increase in cell apoptosis when cells were stimulated by Ox-LDL. In contrast, normal HUVECs adhered, adopted a spindle or pentagonal shape, and proliferated at normal rates (Figure $1 \mathrm{~A}$ and $\mathrm{B}$ ). Cell proliferation also decreased with increased concentrations of Ox-LDL (Table 1).

\section{Ox-LDL influenced the cell cycle and apoptosis rate of HUVECs}

To further evaluate the effect of endothelial injury, the cell cycle and apoptosis rate were determined by Annexin- $V$ staining. As shown in Figure $2 A$ and $B$, the $S$ phase population of HUVECs incubated with Ox-LDL $(100 \mu \mathrm{g} / \mathrm{mL})$ for $24 \mathrm{~h}$ was significantly greater than in the control group (Table 2), suggesting that cells were blocked in the $S$ phase. In addition, flow cytometry revealed that the apoptosis rate of HUVECs incubated with Ox-LDL was higher than in HUVECs incubated under normal conditions (Figure 3 and Table 2).

\section{Ox-LDL upregulated OX40L expression via LOX-1}

To quantify the expression of OX40L during atherosclerotic injury in endothelial cells, we constructed a cell 
injury model by stimulating HUVECS with $100 \mu \mathrm{g} / \mathrm{mL}$ OxLDL for $24 \mathrm{~h}$. OX40L protein and mRNA expression in HUVECs were increased compared with expression in the normal control group (Figures $4 \mathrm{~A}, 5 \mathrm{~A}$ and $\mathrm{B}$, and $6 \mathrm{~A}$ ). Furthermore, OX40L was expressed on HUVEC cell membranes (Figure 5).

To explore the role of LOX-1 in the upregulation of OX40L during atherosclerotic endothelial cell injury, we pretreated HUVECs with $250 \mu \mathrm{g} / \mu \mathrm{L}$ Poly I at $1 \mathrm{~h}$ to partially block the LOX-1 receptor (15). Then, $100 \mu \mathrm{g} / \mathrm{mL}$ Ox-LDL was added to the HUVECs for $24 \mathrm{~h}$. Following these treatments, OX40L mRNA and protein decreased compared with the untreated group (Figures 4A, 5B and $\mathrm{C}$, and $6 \mathrm{~A}$ ). The results of the OX40L assay are shown in Figures 4B, 5D, and 6B.

To confirm that Poly I produced its effects by blocking the LOX-1 receptor, we incubated HUVECs for $24 \mathrm{~h}$ under the following conditions: 1) no Ox-LDL, 2) $100 \mu \mathrm{g} / \mathrm{mL}$ Ox-LDL, and 3) $250 \mu \mathrm{g} / \mu \mathrm{L}$ Poly I plus $100 \mu \mathrm{g} / \mathrm{mL}$ Ox-LDL. The results showed that LOX-1 protein expression in group 2 increased compared to group 1, and LOX-1 protein in group 3 decreased compared with group 2 (Figure 7).

\section{Discussion}

The goal of this study was to determine whether Ox-LDL stimulated the expression of OX40L in HUVECs via a LOX-1-dependent mechanism. The major findings of this study were: 1) Ox-LDL significantly suppressed HUVEC proliferation, and the cells were arrested in the $S$ phase of

A
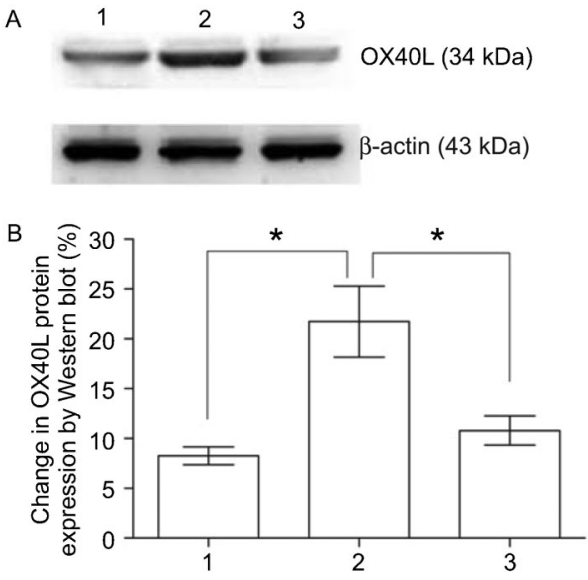

Figure 4. $A, \mathrm{OX} 40 \mathrm{~L}$ expression levels in HUVECs treated with 1) no Ox-LDL (untreated control); 2) $100 \mu \mathrm{g} / \mathrm{mL}$ Ox-LDL, and 3) $250 \mu \mathrm{g} / \mu \mathrm{L}$ Poly I plus $100 \mu \mathrm{g} / \mathrm{mL}$ Ox-LDL. B, The expression of OX40L in group 2 was increased compared with group 1, while OX40L protein expression in group 3 was decreased compared with group 2 ( $n=4 ;{ }^{*} \mathrm{P}<0.05, t$-test). HUVECs: human umbilical vein endothelial cells; Ox-LDL: oxidative low-density lipoprotein; Poly I: polyinosinic acid. the cell cycle; 2) stimulation with Ox-LDL increased OX40L protein and mRNA expressions in endothelial cells; and 3) this increase was inhibited partially by blocking LOX-1 with Poly I. Together, these results suggest that Ox-LDL regulates OX40L expression through a LOX-1-mediated mechanism. Furthermore, the inhibition of HUVEC proliferation and stimulation of apoptosis by Ox-LDL, which is closely related to the progression of atherosclerosis, may be affected by the expression of OX40L.

OX40, which is a type I transmembrane glycoprotein, and $\mathrm{OX} 40 \mathrm{~L}$, which is a type II transmembrane glycoprotein, are costimulatory molecules. OX40L is mainly expressed in mature, antigen-presenting cells, activated B cells, vascular endothelial cells, umbilical vein endothelial cells (HUVEC), and macrophages (16).

LOX-1 shares no structural homology with any other lipoprotein receptor. In addition, LOX-1 can only bind to Ox-LDL and cannot bind to natural LDL or acetylated LDL. Furthermore, LOX-1 can mediate Ox-LDL-related effects, such as vascular endothelial cell dysfunction, foam cell formation, apoptosis, and inflammatory mediator secretion. As such, LOX-1 plays a key role in atherosclerotic plaque formation and development $(17,18)$.

In this study, when HUVECs were cultured with OxLDL, OX40L protein and mRNA expression increased markedly compared with the control group. In contrast, OX40L mRNA and protein expression decreased when HUVECs were pretreated with Poly I, a partial LOX-1 receptor inhibitor. These results show that Ox-LDL upregulates OX40L in HUVECs and that this effect is mediated by LOX-1. Blocking the LOX-1 receptor may therefore be a viable method to reduce the inflammatory response in atherosclerosis.

This study showed, for the first time, the relationship between Ox-LDL and OX40L expression in HUVECs. Moreover, we found that LOX-1 mediated a specific cell signal transduction pathway. These results highlight the important role of LOX-1 in the regulation of OX40L expression during endothelial injury, indicating that LOX-1 may also play a role in the development of atherosclerosis by altering endothelial cell function.

Ox-LDL has additional targets and functions. When Ox-LDL combines with LOX-1, the nuclear transcription

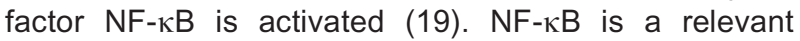
transcription factor for a variety of inflammatory mediators. Our findings indicate that an increase in OX40L expression mediated by LOX-1 may be an intermediate link in the inflammatory response that occurs during the development of atherosclerotic plaque. Future studies are needed to examine the effects of $N F-\kappa B$ activation, which occurs downstream of LOX-1. Nonetheless, reducing the secretion of inflammatory factors by blocking or limiting LOX-1 expression on the surfaces of endothelial cells in atherosclerotic plaques may be a novel drug treatment target for atherosclerosis and may provide new clinical treatment strategies for acute coronary syndrome. 

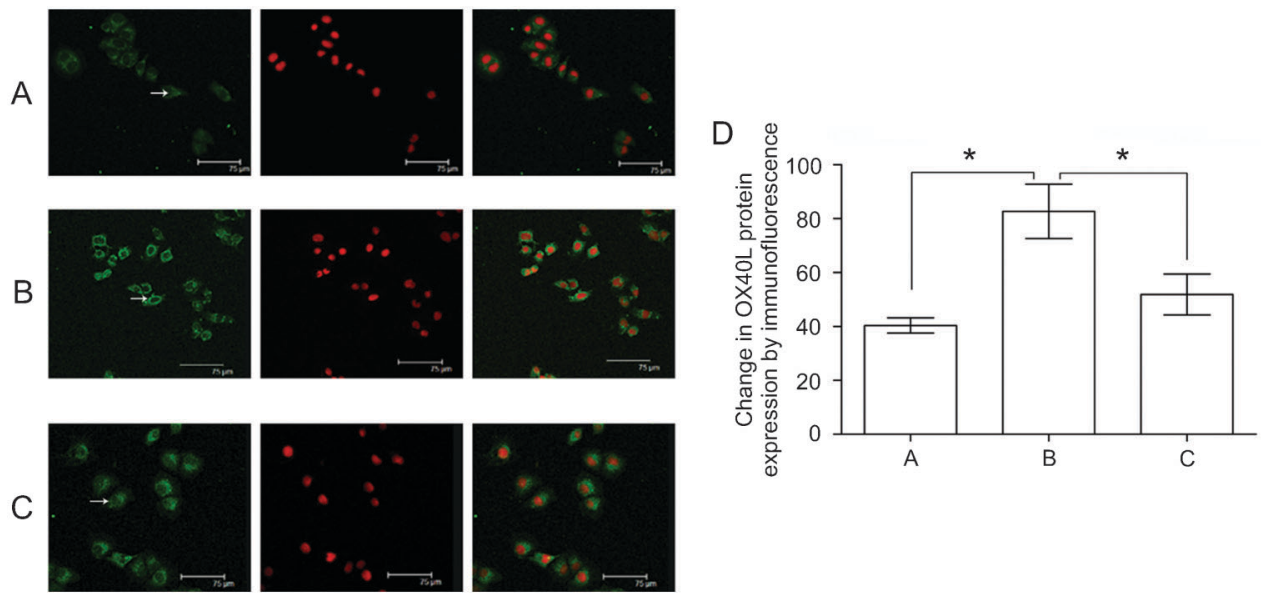

Figure 5. Change in OX40L expression with $A$, no Ox-LDL; $B, 100 \mu \mathrm{g} / \mathrm{mL}$ Ox-LDL; $C, 250 \mu \mathrm{g} / \mu \mathrm{L}$ Poly I plus $100 \mu \mathrm{g} / \mathrm{mL}$ Ox-LDL. The cells were observed by laser confocal microscope $(b a r=75 \mu \mathrm{m})$. Green fluorescence indicates OX40L protein. Red fluorescence indicates nucleolus. Red and green fluorescence show the location and volume of OX40L protein. $D$, Average fluorescence value of 8 randomly selected cells. OX40L was expressed on HUVEC membranes. OX40L protein expression in group B was increased compared to group $A$, while the expression of OX40L in group $C$ was decreased compared to group $B\left(n=4 ;{ }^{*} P<0.05, t\right.$-test). HUVECs: human umbilical vein endothelial cells; Ox-LDL: oxidative low-density lipoprotein; Poly I: polyinosinic acid.
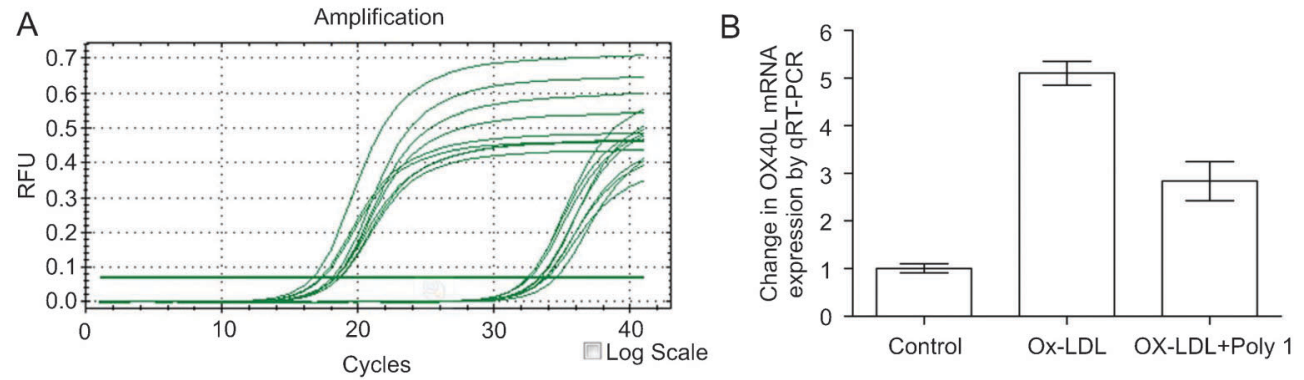

Figure 6. A, Quantification cycles of OX40L mRNA. B, OX40L expression with no Ox-LDL (Control), $100 \mu \mathrm{g} / \mathrm{mL}$ Ox-LDL, and $250 \mu \mathrm{g} / \mu \mathrm{L}$ Poly I plus $100 \mu \mathrm{g} / \mathrm{mL}$ Ox-LDL. OX40L mRNA expression in Ox-LDL was increased compared to control while OX40L mRNA expression in Ox-LDL + Poly 1 was decreased compared to Ox-LDL $\left(n=4 ;{ }^{*} \mathrm{P}<0.05\right.$, $t$-test). Ox-LDL: oxidative low-density lipoprotein; Poly I: polyinosinic acid.

A

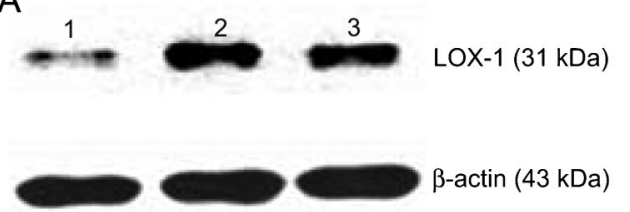

$\mathrm{B}$

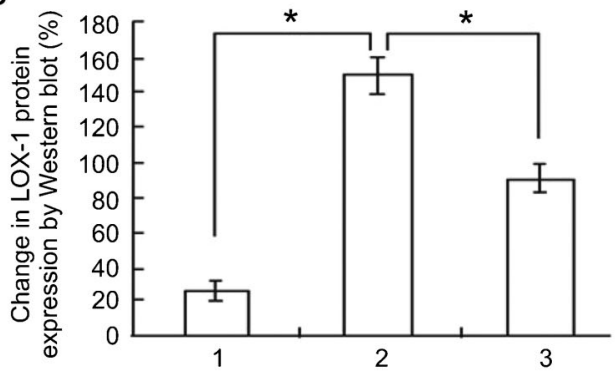

Figure 7. A, LOX-1 expression levels in HUVECs treated with 1) no Ox-LDL (untreated control); 2) $100 \mu \mathrm{g} / \mathrm{mL}$ Ox-LDL, and 3) $250 \mu \mathrm{g} /$ $\mu \mathrm{L}$ Poly I plus $100 \mu \mathrm{g} / \mathrm{mL}$ Ox-LDL. B, The expression of LOX-1 in group 2 was increased compared to group 1 . LOX-1 protein expression in group 3 was decreased compared to group 2 ( $n=4 ;{ }^{*} \mathrm{P}<0.05$, $t$-test). LOX-1: oxidized low-density lipoprotein-1 receptor; HUVECs: human umbilical vein endothelial cells; Ox-LDL: oxidative low-density lipoprotein; Poly I: polyinosinic acid. 


\section{Acknowledgments}

We wish to thank Professor Kang-Hua Ma, Dr. Su-Xin Luo, and Professor Wei-Xue Tang for their helpful discussions and technical assistance in this study. Research supported by the Department of Cardiology, First Affiliated Hospital, Chongqing Medical University, China.

\section{References}

1. Gotsman I, Sharpe AH, Lichtman AH. T-cell costimulation and coinhibition in atherosclerosis. Circ Res 2008; 103: 1220-1231, doi: 10.1161/CIRCRESAHA.108.182428.

2. Ross R. Atherosclerosis - an inflammatory disease. $N$ Engl J Med 1999; 340: 115-126, doi: 10.1056/ NEJM199901143400207.

3. Tousoulis D, Davies G, Stefanadis C, Toutouzas P, Ambrose JA. Inflammatory and thrombotic mechanisms in coronary atherosclerosis. Heart 2003; 89: 993-997, doi: 10.1136/heart.89.9.993.

4. Liuzzo G. Atherosclerosis: an inflammatory disease. Rays 2001; 26: 221-230.

5. Hayashida K, Kume N, Minami M, Kita T. Lectin-like oxidized LDL receptor-1 (LOX-1) supports adhesion of mononuclear leukocytes and a monocyte-like cell line THP-1 cells under static and flow conditions. FEBS Lett 2002; 511: 133-138, doi: 10.1016/S0014-5793(01)03297-5.

6. Wang X, Ria M, Kelmenson PM, Eriksson P, Higgins DC, Samnegard A, et al. Positional identification of TNFSF4, encoding OX40 ligand, as a gene that influences atherosclerosis susceptibility. Nat Genet 2005; 37: 365-372, doi: $10.1038 / n g 1524$.

7. Shi JZ, Wang LY, Zhu Y, Zhao CY, Liu W, Jiang SL, et al. OX40 ligand levels and high-sensitivity $\mathrm{C}$-reactive protein levels in blood from local coronary plaque and the femoral artery in patients with acute coronary syndrome or stable angina. J Int Med Res 2011; 39: 1275-1283, doi: 10.1177/ 147323001103900415.

8. Febbraio M, Hajjar DP, Silverstein RL. CD36: a class B scavenger receptor involved in angiogenesis, atherosclerosis, inflammation, and lipid metabolism. J Clin Invest 2001; 108: $785-791$.

9. Sawamura T, Kume N, Aoyama T, Moriwaki H, Hoshikawa $\mathrm{H}$, Aiba $\mathrm{Y}$, et al. An endothelial receptor for oxidized lowdensity lipoprotein. Nature 1997; 386: 73-77, doi: 10.1038/ 386073a0

10. Murphy JE, Vohra RS, Dunn S, Holloway ZG, Monaco AP, Homer-Vanniasinkam S, et al. Oxidised LDL internalisation by the LOX-1 scavenger receptor is dependent on a novel cytoplasmic motif and is regulated by dynamin-2. J Cell Sci 2008; 121: 2136-2147, doi: 10.1242/jcs.020917.

11. Reiss $A B$, Anwar K, Wirkowski P. Lectin-like oxidized low density lipoprotein receptor 1 (LOX-1) in atherogenesis: a brief review. Curr Med Chem 2009; 16: 2641-2652, doi: 10.2174/092986709788681994.

12. Xu X, Gao X, Potter BJ, Cao JM, Zhang C. Anti-LOX-1 rescues endothelial function in coronary arterioles in atherosclerotic ApoE knockout mice. Arterioscler Thromb Vasc Biol 2007; 27: 871-877, doi: 10.1161/01.ATV.0000259358. 31234.37

13. Mendez-Cruz AR, Paez A, Jimenez-Flores R, Reyes-Reali J, Varela E, Cerbulo-Vazquez A, et al. Increased expression of inflammation-related co-stimulatory molecules by HUVECs from newborns with a strong family history of myocardial infarction stimulated with TNF-alpha and oxLDL. Immunol Lett 2007; 111: 116-123, doi: 10.1016/j.imlet.2007. 06.008.

14. Moriwaki H, Kume N, Sawamura T, Aoyama T, Hoshikawa $\mathrm{H}$, Ochi $\mathrm{H}$, et al. Ligand specificity of LOX-1, a novel endothelial receptor for oxidized low density lipoprotein. Arterioscler Thromb Vasc Biol 1998; 18: 1541-1547, doi: 10.1161/01.ATV.18.10.1541.

15. Wang M, Lu YJ. Effects of lectin-like oxidized low density lipoprotein receptor-1 (LOX-1) on MMP-9 expression in cultured THP-1 monocyte-derived macrophages and the protective effect of simvastatin. J Shandong University (Health Sciences) 2008; 12: 1136-1140.

16. Croft M. Co-stimulatory members of the TNFR family: keys to effective T-cell immunity? Nat Rev Immunol 2003; 3: 609620, doi: $10.1038 /$ nri1148.

17. Mehta JL, Chen J, Hermonat PL, Romeo F, Novelli G. Lectin-like, oxidized low-density lipoprotein receptor-1 (LOX-1): a critical player in the development of atherosclerosis and related disorders. Cardiovasc Res 2006; 69 36-45, doi: 10.1016/j.cardiores.2005.09.006.

18. Ishino S, Mukai T, Kume N, Asano D, Ogawa M, Kuge Y, et al. Lectin-like oxidized LDL receptor-1 (LOX-1) expression is associated with atherosclerotic plaque instability - analysis in hypercholesterolemic rabbits. Atherosclerosis 2007; 195 : 48-56, doi: 10.1016/j.atherosclerosis.2006.11.031.

19. Li D, Mehta JL. Upregulation of endothelial receptor for oxidized LDL (LOX-1) by oxidized LDL and implications in apoptosis of human coronary artery endothelial cells: evidence from use of antisense LOX-1 mRNA and chemical inhibitors. Arterioscler Thromb Vasc Biol 2000; 20: 11161122, doi: 10.1161/01.ATV.20.4.1116. 